\title{
INSIDER WP5 (in situ measurements): developed activities, main results and conclusions
}

\author{
Margarita Herranz ${ }^{1,}{ }^{*}$, Raquel Idoeta ${ }^{1}$, Khalil Amgarou ${ }^{2}$, Frédéric Aspe $^{3}$, Csilla Csöme ${ }^{4}$, \\ Sven Boden ${ }^{5}$, and Marielle Crozet ${ }^{6}$ \\ 1 Nuclear Engineering and Fluid Mechanics dpt., University of the Basque Country (UPV/EHU), Pza. Ingeniero Torres \\ Quevedo 1, 48013 Bilbao, Spain \\ 2 Commissariat à l'énergie atomique et aux énergies alternatives CEA, Direction de l'énergie nucléaire DEN, DDCC/CCMA/ \\ GA2P, Marcoule, BP 17171, 30207 Bagnols sur Cèze Cedex, France \\ 3 Mesures Nucléaires, ONET Technologies, 970 chemin des agriculteurs, 26701 Pierrelatte, France \\ 4 Nuclear Security Department, Centre for Energy Research (EK), Konkoly-Thege M. 29-33,1021 Budapest, Hungary \\ ${ }^{5}$ Dismantling, Decontamination and Waste Expert Group, Belgian Nuclear Research Centre (SCK•CEN), Boeretang 200, \\ 2400 Mol, Belgium \\ ${ }^{6}$ Commissariat à l'énergie atomique et aux énergies alternatives CEA, Direction de l'énergie nucléaire DEN, DMRC, Univ. \\ Montpellier, Marcoule, BP 17171, 30207 Bagnols sur Cèze Cedex, France
}

Received: 14 September 2019 / Received in final form: 19 November 2019 / Accepted: 10 December 2019

\begin{abstract}
Within the INSIDER project, the WP5 (in situ measurements) has been tasked with analysing the existing systems and methodologies for carrying out these types of measurements in constrained environments, aiming to classify and categorise these environments. An additional task is to organise the participation in in situ intercomparison exercises in real situations, defining the most suitable equipment to carry these out. This paper presents the activities of the WP5 and a summary of the main results obtained in these activities after the first two years of work.
\end{abstract}

\section{Introduction}

INSIDER is an EU Horizon 2020 research project, within the topic NFRP-7 of the EURATOM programme that aims to develop and validate a new and improved integrated characterisation methodology and strategy during nuclear decommissioning and dismantling operations (D\&D) of nuclear power plants, post-accidental land remediation of nuclear facilities under constrained environments. In line with the general objectives of the INSIDER project, the work package WP5 is devoted to the definition and implementation of the practical considerations surrounding in situ radiological characterization of nuclear/ radioactive facilities subject to a decommissioning programme, taking into account specific outputs from work packages WP2, WP3 and WP4.

So far, and according to the WP5 work plan as defined at the start of the project, the main activities undertaken and results obtained by this WP are related to: (a) analysis of the different existing measurement systems for the in

\footnotetext{
* e-mail: m.herranz@ehu.eus
}

situ measurement of alpha, beta, neutron and gamma radiations emitted from the materials and structures belonging to radioactive/nuclear installations under D\&D processes in a constrained environment, (b) classification and characterization of this type of environments, based on the restrictions they impose on the measurement system and (c) application of the lessons learned to a practical situation, achieving the first intercomparison exercise carried out in a real installation under D\&D decommissioning process.

The first two of these activities have led to deliverables 5.1: "Inventory of existing methodologies for constrained environments" [1] and 5.2: "Classification and categorization of the constrained environments" [2], while the third one is already finished but the data obtained are still under evaluation by colleagues from WP6, remaining the WP5 in charge of the technical challenges.

At the same time, as these activities have been carried out, it should be noted that a database of the main companies which carry out D\&D activities at the European level has been set up and it will remain operational and open for the duration of the project.

In this paper, the main conclusions obtained during these tasks are briefly presented and analysed. 


\section{Inventory of existing methodologies for constrained environments}

\subsection{Description}

The objective of this work is to describe the instruments used for in situ radiological characterisation by means of non-destructive techniques, taking into account their limitations of use in constrained environments, for example, in terms of radioactivity (medium or high radioactivity), under difficult accessibility conditions and/or in underwater interventions. The most commonly used instruments are described, as well as the new developments. Additionally, this study also describes those instruments that are only used in very specific situations or whose use is not very widespread due to technological, economic or time availability constraints.

This description starts with the simplest, fastest and most inexpensive method that can be used, which is based on measuring radiation levels at predefined locations (environmental radiation measurements). The cartography of alpha/beta contamination on surfaces is also a very useful method and is thus analysed as well (surface contamination measurements). Other, more sophisticated methods considered in the document that can be applied for in situ measurements are gamma spectrometry, passive neutron counting and radiation cameras.

It is important to note, however, that certain nuclear facilities or some of their components contain complex or non-standard infrastructures with limited accessibility and intense radiation fields. For such constrained environments, new methodologies are necessary. These will be based on advanced statistical processing and modelling, coupled with adapted and innovative analytical and measurement methods. Robotics or other remotely deployed systems based on reduced-size detectors are a good alternative, but collimation mechanisms with small opening angles may also be considered to restrict the fieldof-view of the chosen instruments to only specific areas or portions of the item to be measured. But these new or stillunder-development methodologies are beyond the scope of this document.

\subsection{Main results and conclusions}

\subsubsection{Environmental radiation measurements}

The main advantages of the Geiger-Müller (GM), the most widely used instrument for gross beta/gamma counting, are its low price, robustness, its large variety of sizes and a minimal electronic processing. However, the GM cannot distinguish between radiation types or energies, it is not able to measure high dose rates, and sustained high radiation levels will definitively degrade its detection performance, so it is not recommended for certain constrained environments [3].

Ionization chambers are universally used to determine air-kerma, due to their good and uniform response to photons and their tolerance to intense radiation fields. They, however, cannot discriminate between radiation types and cannot provide the corresponding energy spectrum. Another alternative in the case of energetic photons is the one based on the energy-compensated silicon diodes, allowing for very small-sized detectors to be used for routine surveys in nuclear facilities.

Virtually every type of photon detector is able to measure $H^{*}(10)$, but the preferable one is the proportional counter, which is able to discriminate between radiation types and provides the corresponding spectrometric information. It also has a small dead time effect and can tolerate high radiation levels. The case of neutrons is much more complex and the available instruments to measure the associated ambient dose equivalent provide satisfactory results only in restricted energy intervals and/or in specific irradiation conditions. Therefore, such instruments must be calibrated under the same experimental configurations, or at least considering a representative neutron spectrum [4].

\subsubsection{Surface contamination measurements}

For alpha contamination, gas-filled proportional counters or scintillator detectors with an ultra-thin aluminized Mylar or mica film window can be used, positioning the counter as close as possible to the object under analysis. However, the risk of breakage makes direct measurements impractical, except in particular and controlled situations. In the case of $\beta$-particles, the use of proportional counters with more robust end-window thickness is more widespread. Nevertheless, beta identification on the basis of energy resolution is virtually impossible and care must be taken to properly estimate the contribution of gamma radiation.

\subsubsection{Gamma spectrometry}

Gamma spectrometry is the start technique for in situ measurements, allowing for the identification and even the quantification of most radionuclides [5]. The most commonly used gamma spectrometers are based on inorganic scintillators, such as $\mathrm{NaI}(\mathrm{Tl})$ or $\mathrm{LaBr}_{3}(\mathrm{Ce})$, as well as on high-purity germanium (HPGe), CdTe or CdZnTe (CZT) semiconductors.

Scintillation detectors can be manufactured in large volumes, and they have a high detection efficiency, but a poor energy resolution. They are often used for low intensity photon flux measurements with simple gamma spectra. However, since most scintillators have a very fast signal response they can also be used at high counting rates or for coincidence counting. $\mathrm{NaI}(\mathrm{Tl})$ scintillation detectors do not tolerate high radiation levels, and they are hygroscopic. Consequently, they cannot tolerate exposure to humid environments, so they must be hermetically sealed, which can hinder low energy gamma detection. These detectors provide a stable energy resolution and a constant decay time of the light pulses over a wide range of temperatures [6]. Once hermetically sealed, they can also be used in underwater applications, inside a nuclear fuel storage pool for real-time monitoring. $\mathrm{LaBr}_{3}(\mathrm{Ce})$ scintillators offer better energy resolution than $\mathrm{NaI}(\mathrm{Tl})$ ones, a fast emission rate, an excellent temperature tolerance, as well as good resistance to intense radiation fields [7]. As they are currently available in small sizes, they are very appropriate for constrained environ- 

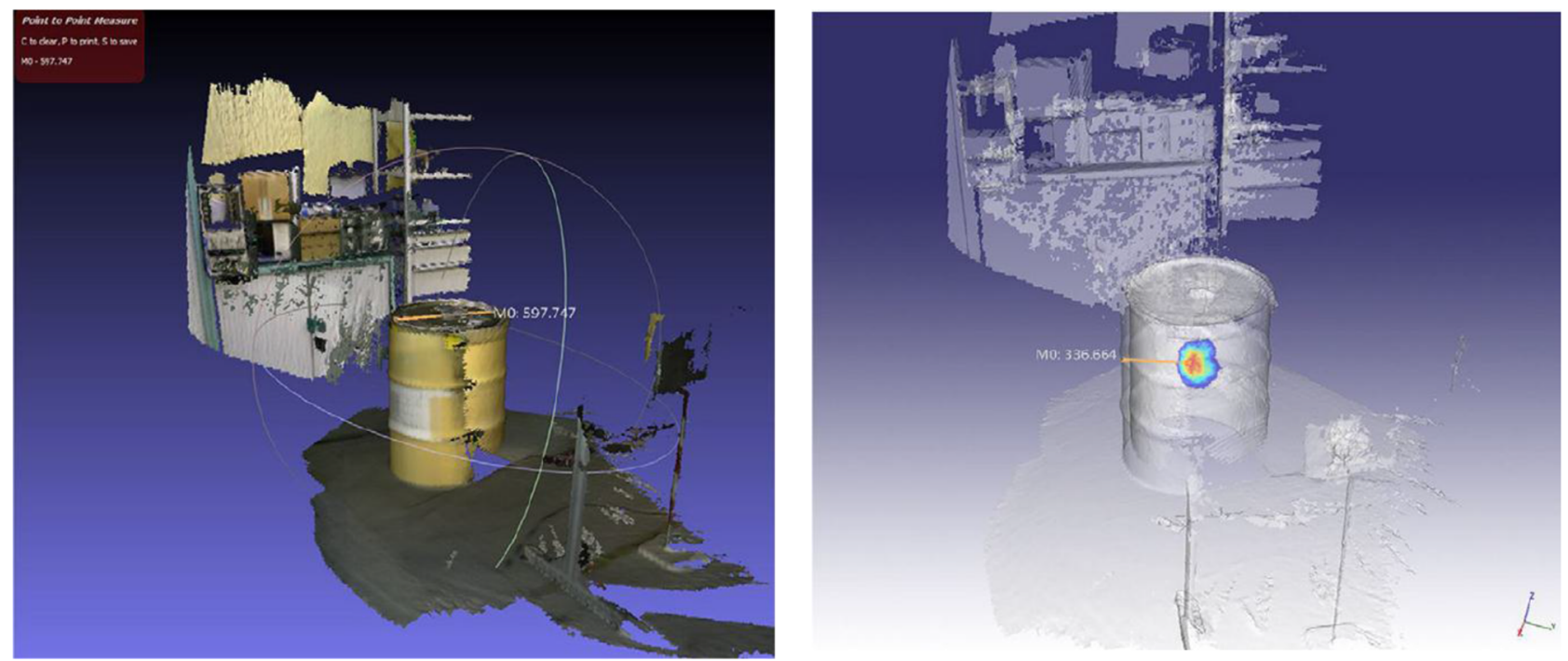

Fig. 1. Illustration of the capability of a stereo $\gamma$-camera to locate a ${ }^{137} \mathrm{Cs}$ source placed inside a barrel with an outer diameter of $60 \mathrm{~cm}$, taken from Paradiso et al. [9].

ments, just like silicon drift detectors (SDD) and silicon photomultipliers (SiPM).

High-purity Germanium (HPGe) detectors have an excellent energy resolution and they may have large sensitive volumes. Their major drawback is that the crystal must be cooled to liquid nitrogen temperatures, which limits their usefulness. CdTe and CZT detectors have higher detection efficiency than HPGe ones although it is difficult to obtain them in large sizes, and they can operate at room temperatures. Their energy resolution is not as optimal as that of HPGe detectors, but it is slightly better than that of scintillators. In addition, CdTe and CZT detectors are able to carry out measurements over a wide range of radiation levels; however, they are characterized by a low-energy tailing in the measured spectrum.

It must be highlighted that semiconductor detectors are relatively sensitive to performance degradation when exposed to intense radiation fields, namely, the ones containing neutrons. Electronic components are also radiation sensitive, particularly the preamplifiers.

\subsubsection{Neutron coincidence measurements}

The main advantage of passive neutron measurement is its relatively low sensitivity to the density of the materials surrounding the radioactive elements. However, it is extremely affected by a number of frequently unknown properties, such as the presence in the sample of ${ }^{242} \mathrm{Cm}$ and ${ }^{244} \mathrm{Cm}$.

The main disadvantage of its basic mode of application is its high sensitivity to the chemical form of the radioactive contaminant, thus it is necessary to discriminate the signal fraction originating from the spontaneous fissions from that one resulting from $(\alpha, n)$ reactions. A precise interpretation of the results requires prior knowledge of the isotopic composition of the contaminant. Failing this, only an overall assessment representing all the potential emitting isotopes will be possible.

\subsubsection{Laser-induced breakdown spectroscopy and radiation cameras}

All these techniques are quite promising and, although they are not widely used in the nuclear industry, they have the potential to become standard procedures in this field in the near future. In what follows, we provide a brief description of each one of them.

Laser-induced breakdown spectroscopy or LIBS is considered to be a minimally-destructive assay method based on the principle of ablation of a small amount of sample $\left(10^{-12}\right.$ to $\left.10^{-9} \mathrm{~g}\right)$ by focusing a highly energetic laser pulse onto a given surface point. The ablated material then forms a micro-plasma, which almost immediately emits light photons at characteristic wavelengths, depending on the elemental composition of the sample. It is therefore a very fast and versatile technique that can, in principle, detect all kind of materials, including impurities. However, its application to the radiological characterization field is quite limited because this system is unable to distinguish radioisotopes.

Gamma imaging techniques enable the superimposition of a colour map display, indicating the amount of emitted X- or $\gamma$-rays, on a given optical image of the scene under study. It provides an optimal solution to track most radioactive sources from greater distances than conventional rate meters, thus significantly reducing the radiation dose received by operators. $\gamma$-cameras for industrial applications have recently undergone impressive upgrades in terms of lightness, compactness, usability, response sensitivity, angular resolution and spectrometric capabilities [8]. In this regard, perhaps the main technological breakthrough has so far been the development of a stereo $\gamma$-camera [9], which is able to automatically retrieve the 3 -D location of any radioactive source, regardless of its shape and volume, even when this source is behind or within an occluding object, as shown in Figure 1. 


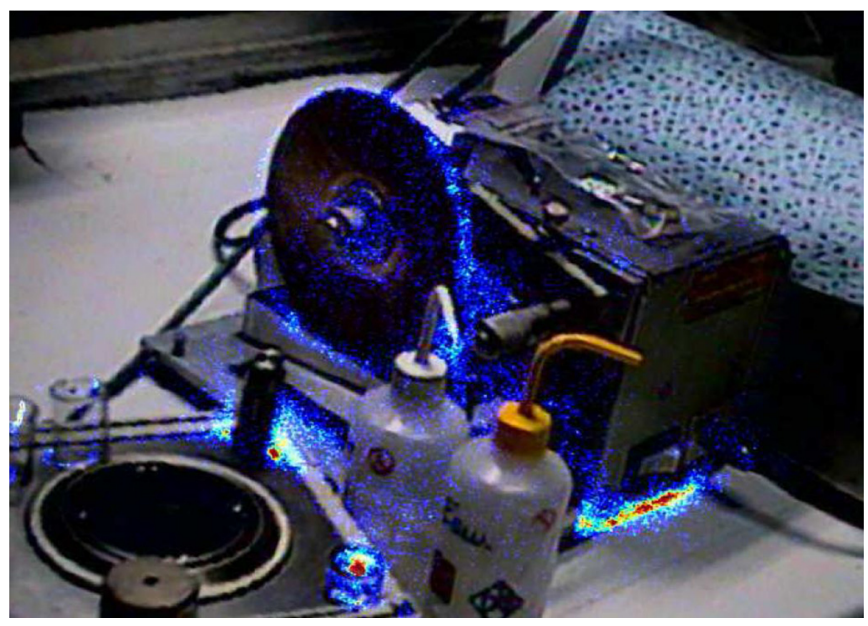

Fig. 2. Localization by means of an alpha camera of plutonium contamination inside a glove box of the ATALANTE facility at the CEA Marcoule site.

In principle, the difference between neutron imaging and gamma cameras is the type of materials used for shielding. Several prototypes have recently been developed for multiple applications related to the radiation protection of workers, and the protection of nuclear non-proliferation safeguards and national security. However, the challenge for the initial characterisation of nuclear facilities subject to a decommissioning programme remains the design of neutron cameras that are as compact and robust as possible, so that they can be used in constrained environments while remaining sufficiently sensitive to neutrons and optimizing the angular resolution. Potentially good compromises in this aspect have been proposed by Whitney et al. [10] and Lynde et al. [11].

The remote and safe localisation of materials or surfaces contaminated with $\alpha$-particles emitters are possible based on the ionization-induced fluorescence of airborne molecules. In fact, after depositing their energy in a small layer of air, monochromatic ultraviolet lights are emitted because of the presence of nitrogen. This is the measurement basis of an alpha camera, which has been widely tested in realistic fields with encouraging results [12]. As an example, Figure 2 shows how this technique has been successfully applied to locate the surface contamination of the ATALANTE facility at the CEA Marcoule site.

\section{Classification and categorization of the constrained environments}

\subsection{Description}

This work involved the description and categorisation of constrained environments and the identification of the type of nuclear/radioactive installations where they could appear, depending on the D\&D process stage under development.

For a specific component inside a certain installation, the choice of an in situ measurement technique will depend on a set of information obtained through a complete decision process that starts by defining the investigation objectives of the in situ measurement, and finishes by choosing the most suitable investigation method, that is, the most appropriate in situ measurement technique. The selection of that investigation methodology depends on various challenges to be overcome or constraints to be taken into account.

On the basis of the preliminary information gathered, considering the zones of interest along with the existing constrained environment, it is possible to determine the adequate number of in situ measurements, their locations and the equipment needed. This process defines the in situ measurement method - "system definition"; see Figure 3.

To complete this investigation method, the response protocol - "intervention definition"- will be defined by considering challenges like the availability of resources and other considerations related to safety, radiation protection, security and quality.

The output of these processes will be the assessment of the most suitable in situ measurement technique for a specific area under different constraints.

The description of constrained environments includes all types of environments that make the choice of a nondestructive in situ measurement method challenging, as previously described. They can be classified according to the following problems: (a) radioactivity levels of the area to be characterized, (b) difficult accessibility of this area, (c) diversification of the type and properties of the materials contained in it, and (d) the possible presence of chemical and/or biological hazards. A more exhaustive description of the constraints can be seen in Figure 4.

This classification has been accomplished by listing the most important installations where in situ measurements could be taken during the $\mathrm{D} \& \mathrm{D}$ process activities: reactors (power-generating and research), plants (uranium enrichment, fuel converting, fabricating, spent fuel processing, other fuel facilities, radioelement production, nuclear maintenance workshop, storage facilities, low-level radwaste facilities), high energy accelerators and other types of installations (irradiation facilities, testing and research laboratories). Afterwards, we considered that each of them can comprise various areas that can be affected by different constraints in different ways. We consider that these areas can be grouped into 33 categories (e.g. foundationsstructural materials and apron; secondary cooling system; ventilation ducts; decontamination room...).

However, it should be taken into account that a same area can have different constraints throughout the whole decommissioning process. The way that characterization must be carried out depends on the following three phases of any D\&D programme [13]:

- initial - Dismantling phase;

- intermediate - Remediation phase;

- final - Release phase.

Finally, the classification has been accomplished by producing several tables, one for each constraint, where areas are linked to installations and to the specific properties of the constraints. For each one of these constraints, the differences between the various phases 


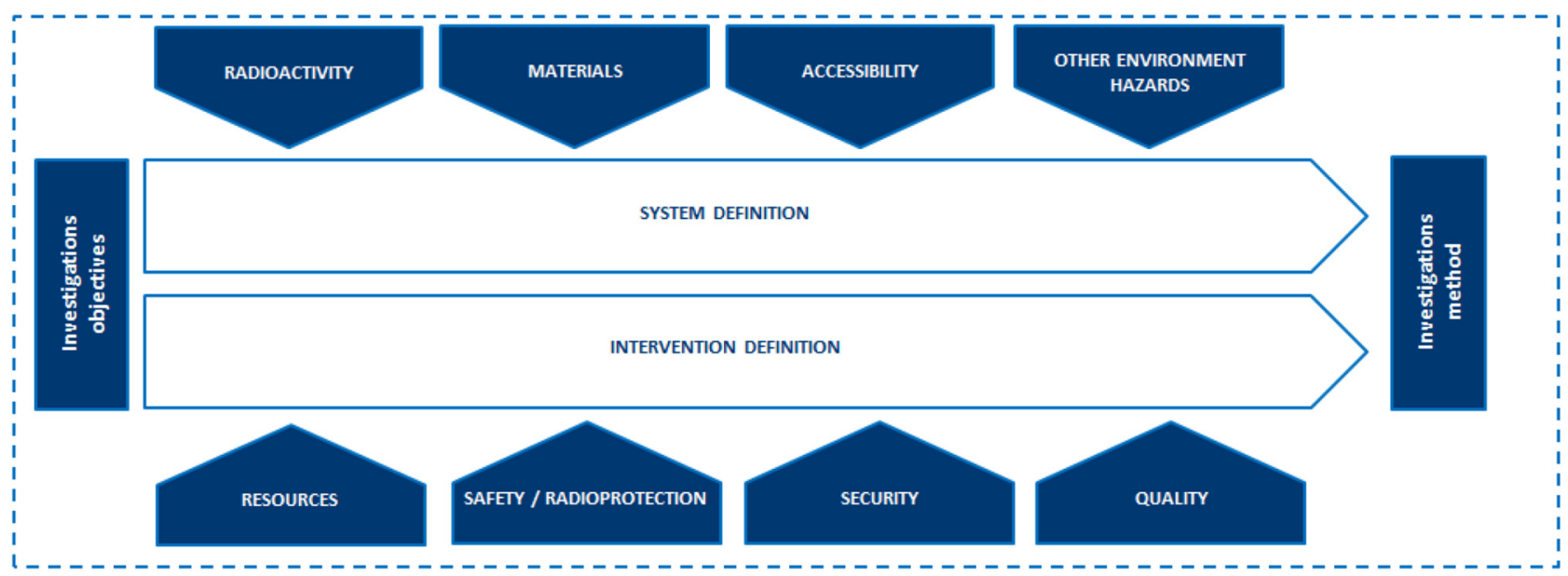

Fig. 3. From Investigation objective to investigation methods.

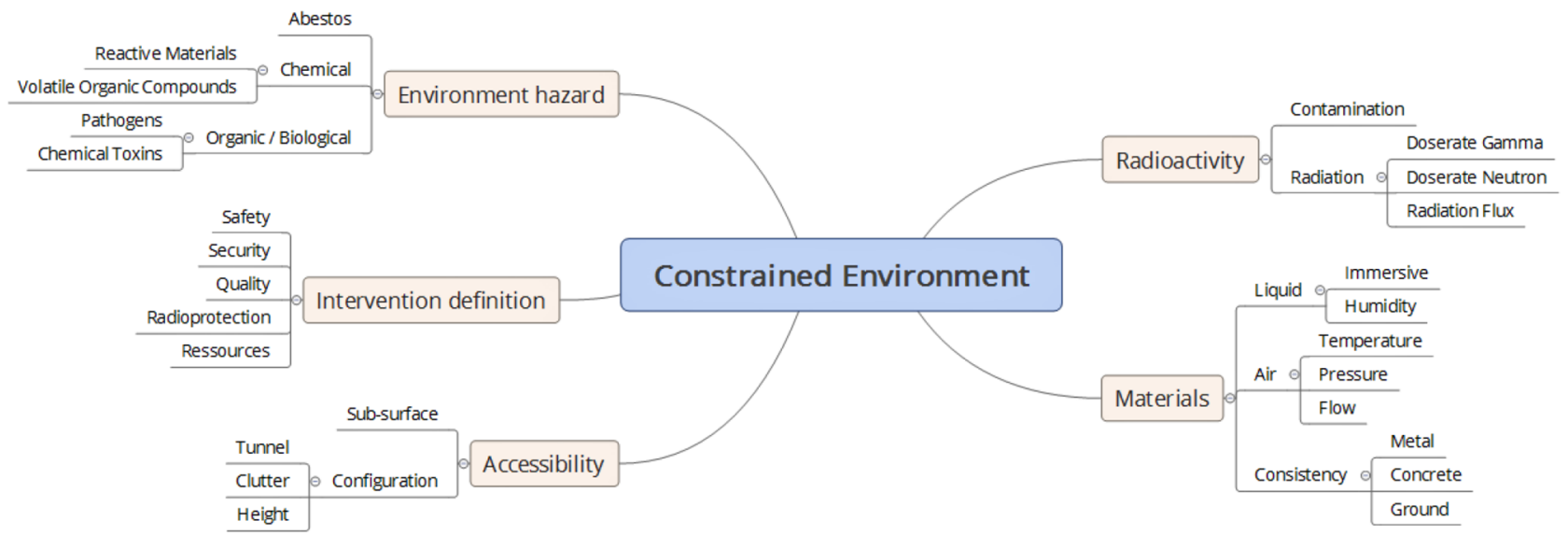

Fig. 4. Constrained environments.

of a given D\&D programme have also been outlined. In the case of radiological environments, the constraints have been categorized as very low, low, normal, high and very high.

This work does not claim to be an exhaustive guide containing all the possible nuclear or radioactive installations, nor all possible constraint environments. The main objective of this document is to describe those situations that appear most often and those that are most challenging. This paper presents only the main conclusions of the study.

\subsection{Main results and conclusions}

Due to space limitations, all tables cannot be presented here, but they are available on the EU INSIDER project website. As an example, Table 1 shows how areas can be classified according to their radioactivity constraints.

As a general conclusion, and as expected, it is in the nuclear power plants where the number of constraints and their categorization is the highest. Therefore, the need to have well-defined methodologies and in situ equipment is more challenging.

\section{Development of the first in situ intercomparison campaign}

\subsection{Description}

Out of the three in situ intercomparison campaigns planned as part of the European project INSIDER, the first one has already finished. This first exercise has been carried out by carrying out radiological measurements in the biological shield of the BR3 reactor, located in the SCKCEN (Mol, Belgium) and under D\&D process. Figure 5 shows a 3D model of the reactor pit (left) and a picture of the platform installed for the measurements (right). The material is quite well characterized and the list of radionuclides it contains includes: ${ }^{3} \mathrm{H},{ }^{14} \mathrm{C},{ }^{41} \mathrm{Ca},{ }^{55} \mathrm{Fe}$, ${ }^{60} \mathrm{Co},{ }^{63} \mathrm{Ni},{ }^{133} \mathrm{Ba},{ }^{134} \mathrm{Cs},{ }^{137} \mathrm{Cs},{ }^{152} \mathrm{Eu},{ }^{154} \mathrm{Eu}$ and ${ }^{155} \mathrm{Eu}$.

At three different points of the biological shielding with high, medium and low dose rates, three types of measures have been carried out: dose rate, total gamma and gamma spectrometry. Due to space and time constraints, only 6 work teams from 7 different organizations were allowed to participate in this intercomparison exercise. 
Table 1. Classification of areas according to the radioactivity constraint (an example).

\begin{tabular}{|c|c|c|c|c|c|c|}
\hline Areas & Type of installation & Step & Contamination & $\begin{array}{l}\text { Gamma } \\
\text { dose rate }\end{array}$ & $\begin{array}{l}\text { Neutron } \\
\text { dose rate }\end{array}$ & $\begin{array}{l}\text { Radiation } \\
\text { flux } \\
\end{array}$ \\
\hline Equipment room & $\begin{array}{l}\text { Reactors } \\
\text { Plants } \\
\text { Accelerators }\end{array}$ & $\begin{array}{l}\text { Intermediate } \\
\text { Final }\end{array}$ & Low & Low & Low & Low \\
\hline Process control room & $\begin{array}{l}\text { Reactors } \\
\text { Plants } \\
\text { Accelerators }\end{array}$ & Final & No & No & No & No \\
\hline $\begin{array}{l}\text { Decontamination } \\
\text { room }\end{array}$ & $\begin{array}{l}\text { Reactors } \\
\text { Plants } \\
\text { Accelerators }\end{array}$ & $\begin{array}{l}\text { Initial } \\
\text { Intermediate } \\
\text { Final }\end{array}$ & High & Low & Low & No \\
\hline Hot Cells front area & $\begin{array}{l}\text { Reactors } \\
\text { Plants } \\
\text { Accelerators }\end{array}$ & $\begin{array}{l}\text { Intermediate } \\
\text { Final }\end{array}$ & Low & No & No & No \\
\hline Refuelling cavity & $\begin{array}{l}\text { Reactors } \\
\text { Plant }\end{array}$ & $\begin{array}{l}\text { Initial } \\
\text { Intermediate } \\
\text { Final }\end{array}$ & $\begin{array}{l}\text { Very high } \\
\text { High }\end{array}$ & $\begin{array}{l}\text { Very high } \\
\text { High }\end{array}$ & $\begin{array}{l}\text { Very high } \\
\text { High }\end{array}$ & No \\
\hline
\end{tabular}
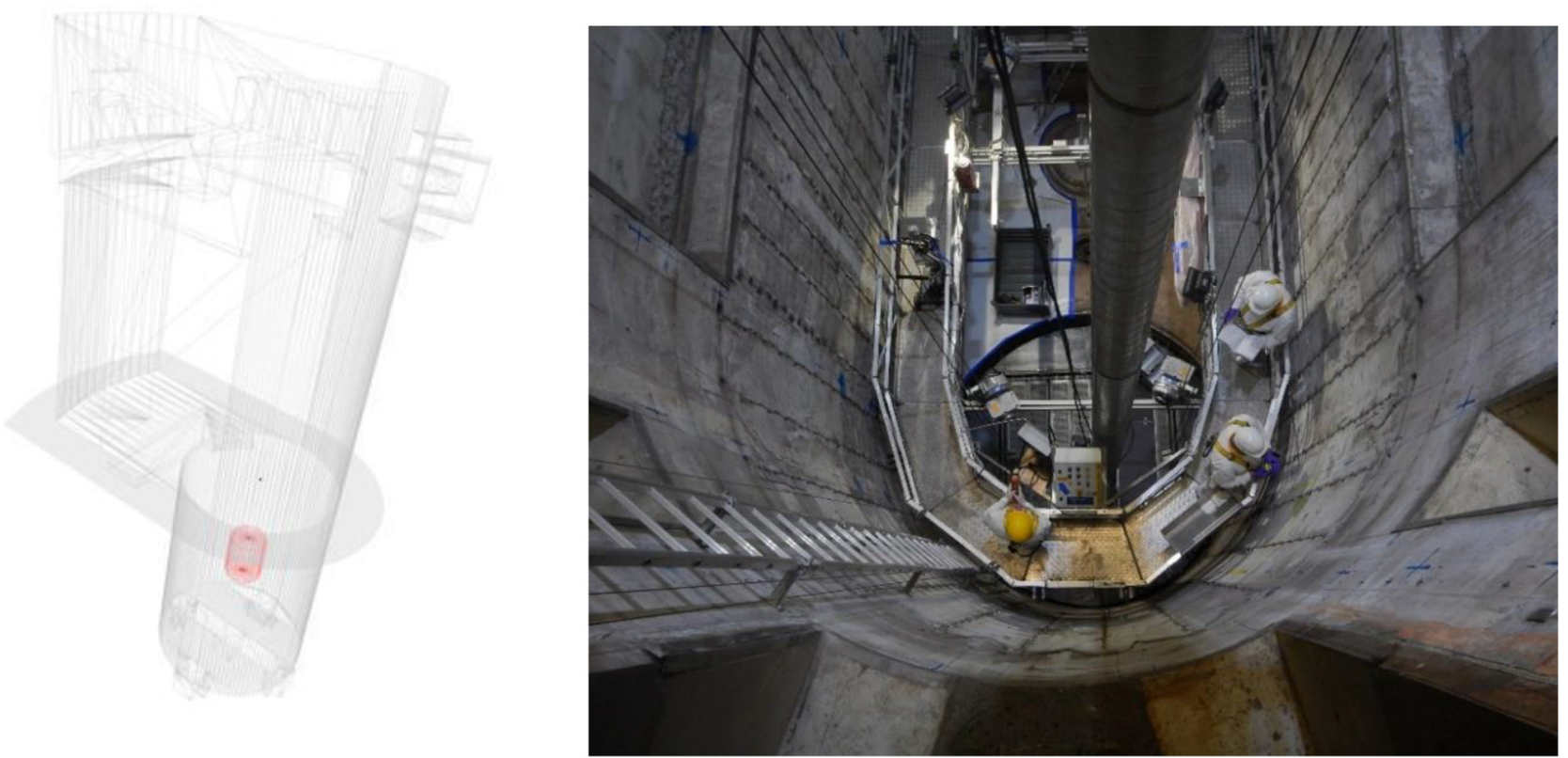

Fig. 5. Reactor pit and platform.

\subsubsection{Dose rate and total gamma determinations}

In this case, 5 rounds with 5 consecutive measurements each were carried out. Between rounds, the equipment was removed and repositioned, so a total of 25 measurements have been taken at each point.

Detectors were neither shielded nor collimated and the distance source-detector was chosen near to zero (i.e. direct contact).

Dose-rate probes were calibrated by each team using their own procedure and considering a ${ }^{137} \mathrm{Cs}$ source; data were provided in terms of ambient dose equivalent rate $\mathrm{H}^{*}(10)$ (in microSv/h). Different probes were used: GM; ionization chambers and scintillators $(\mathrm{CsI}(\mathrm{Tl})$ and $\mathrm{BGO}$ ).
Total-gamma probes were calibrated in situ by using a ${ }^{137} \mathrm{Cs}$ reference source made available by SCK/CEN, with the aim of being able to compare the results from the different counters. Different probes were used: proportional counters and different scintillators ( $\mathrm{ZnS}, \mathrm{NaI}, \mathrm{BGO}$, $\mathrm{LaBr}_{3}$ and plastic ones).

\subsubsection{Gamma spectrometry measurements}

All the participants performed their measurements at the same location, at the bottom of the pool with a higher dose rate, using collimated $\left(90^{\circ}\right)$ and shielded detectors. Each group took two spectra, one with the open collimator and the other with the closed collimator for background spectrum, focusing on a point of the biological shielding, 


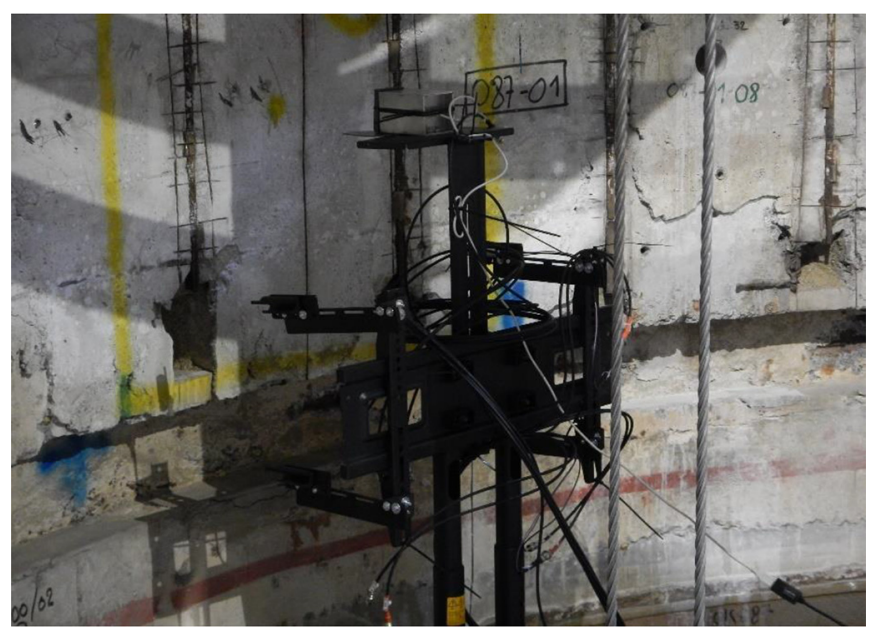

Fig. 6. Gamma spectrometry equipment at position.

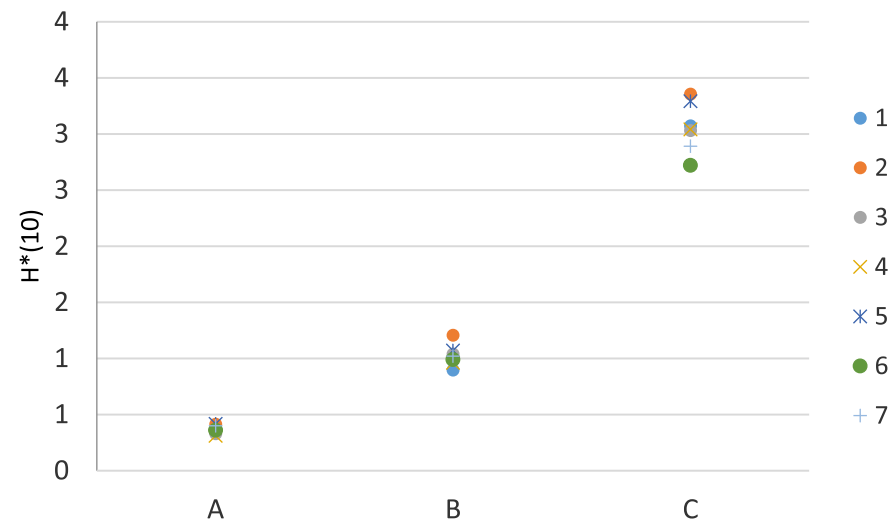

Fig. 7. Ambient dose equivalent rate $H^{*}(10) H^{*}(10)$, in microSv/h, measured in points $\mathrm{A}, \mathrm{B}$ and $\mathrm{C}$ by the 7 different equipments used.

defined beforehand, and at a fixed distance between the detector and the pool wall of $30 \mathrm{~cm}$ (see Fig. 6).

All the teams used their own equipment, as well as their own calibration procedure and method (using different Monte-Carlo codes), in order to check as many of them as possible. Different detectors were used: $4 \mathrm{HPGe}$, one CZT and one $\mathrm{LaBr}_{3}$ were used.

Results expected for the following parameters and in the following units were:

\begin{tabular}{ll}
\hline Depth where ${ }^{133} \mathrm{Ba}$ concentration $<=0.1 \mathrm{~Bq} / \mathrm{g}$ & $\mathrm{m}$ \\
Depth where ${ }^{152} \mathrm{Eu}$ concentration $<=0.1 \mathrm{~Bq} / \mathrm{g}$ & $\mathrm{m}$ \\
Depth where $\mathrm{SUM}\left({ }^{133} \mathrm{Ba} / 0.1+{ }^{152} \mathrm{Eu} / 0.1\right) \mathrm{Bq} / \mathrm{g}<1$ & $\mathrm{~m}$ \\
${ }^{152} \mathrm{Eu} /{ }^{154} \mathrm{Eu}$ activity ratio & $\%$ \\
${ }^{137} \mathrm{Cs}$ surface activity concentration & $\mathrm{Bq} / \mathrm{m}^{2}$ \\
\hline
\end{tabular}

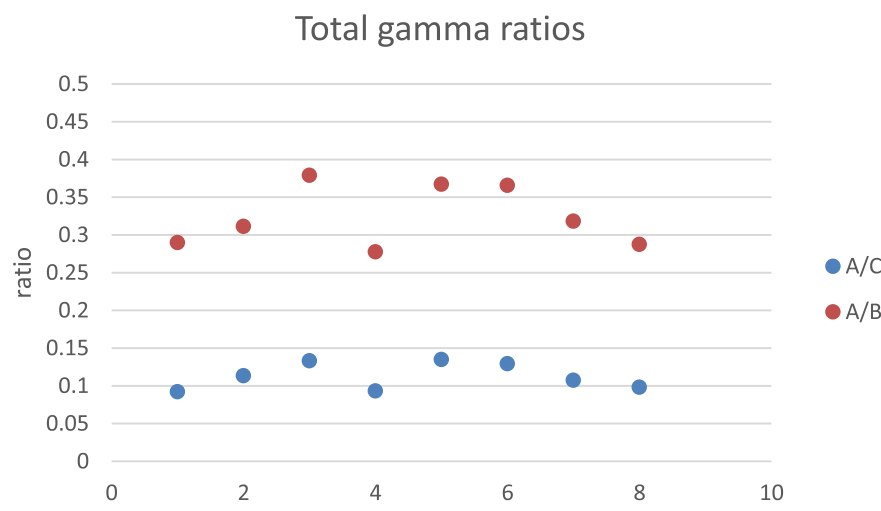

Fig. 8. Total gamma relative values, showing ratios between results from points $\mathrm{A}$ and $\mathrm{C}$ and $\mathrm{A}$ and $\mathrm{B}$ by the 8 different equipment used.

\subsection{Main results and conclusions}

This exercise represents the first intercomparison of onsite measurements in a reactor under decommissioning, which is a milestone in itself. Additionally, this exercise has also been carried out in an environment that is restrictive for measurements. The results are now under statistical analysis however some main conclusions can be outlined.

Regarding the results obtained for the dose-rate, all of them are quite similar and there are no differences that can be attributed to the type of equipment used (see Fig. 7 ). There are differences in terms of uncertainties, but they do not depend on the equipment used but on their active volumes. Two of the most significant challenges of these measurements, the constrained environment with radiation arriving from all points and the time needed to stabilize the equipment between measurements, do not seem to have affected the quality of the measurements.

For total gamma measurements, as expected, there is an enormous dispersion in the results in all points. This dispersion is not really related to the type of detector used, but to the specific configuration of each detector, the different response of each detector to photons depending on their energy, as well as to the beta radiation and to the methodologies used by each team. Consequently, the total gamma measurements cannot be compared from the point of view of the absolute values obtained. However, the relative values obtained from the 3 points measured show more similar results (see Fig. 8).

As for gamma spectrometry measurements, in the case of medium to high concentrations of radionuclides, the results are also consistent. However, for low concentrations, spectrometric measurements are very dependent on the type of equipment, in terms of resolution and efficiency, as well as on the evaluation systems used.

The project INSIDER has received funding from the Euratom research and training program 2014-2018, under grant agreement No 755554. 


\section{Author contribution statement}

K. Amgorou, M. Herranz, C. Csöme and F. Aspe, provided the information for Section 2; F. Aspe and R. Idoeta, provided the information for Section 3; S. Boden. M. Crozet and M. Herranz were responsible of the organization and the realization of the intercomparison campaign. M. Herranz took the lead in writing the manuscript supported by S. Boden. All authors provided critical feedback and helped shape the research, analysis and manuscript.

\section{References}

1. INSIDER. Deliverable 5.1: Inventory of existing methodologies for constrained environments, available at http:// insider-h2020.eu

2. INSIDER. Deliverable 5.2: Classification and categorization of the constrained environments, available at http://insiderh2020.eu

3. G.F. Knoll, Radiation Detection and Measurement, 4th edn. (John Wiley \& Sons, New Jersey, 2010)

4. IAEA, Compendium of neutron spectra and detector responses for radiation protection purposes. International Atomic Energy Agency, Supplement to Technical Reports Series No. 318, 2001, Vienna, available at: http://www-pub. iaea.org/MTCD/Publications/PDF/TRS403_scr.pdf

5. B. Pérot, F. Jallu, C. Passard, O. Gueton, P.G. Allinei, L. Loubet, N. Estre, E. Simon, C. Carasco, C. Roure, L. Boucher, H. Lamotte, J. Comte, M. Bertaux, A. Lyoussi, P. Fichet, F. Carrel, The characterization of radioactive waste: a critical review of techniques implemented or under development at CEA, France, EPJ Nucl. Sci. Technol. 4, 3 (2018)
6. M. Moszynski, A. Nassalski, A. Syntfeld-Kazuch, T. Szczensniak, W. Czarnacki, D. Wolski, G. Pausch, J. Stein, Temperature dependences of $\mathrm{LaBr}_{3}(\mathrm{Ce}), \mathrm{LaCl}_{3}(\mathrm{Ce})$ and $\mathrm{NaI}$ (Tl) scintillators, Nucl. Instrum. Methods Phys. Res. Sect. A, 588, 739 (2006)

7. G. Bizarri, J.T.M. de Haas, P. Dorenbos, C.W.E. van Eijk, Scintillation properties of $\varnothing 1 \times 1 \mathrm{Inch}^{3} \mathrm{LaBr}_{3}$ : $5 \% \mathrm{Ce}^{3+}$ crystal, IEEE Trans. Nucl. Sci. 53, 615 (2006)

8. K. Amgarou, V. Paradiso, A. Patoz, F. Bonnet, J. Handley, P. Couturier, F. Becker, N. Menaa, A comprehensive experimental characterization of the iPIX gamma imager, J. Instrum. 11, P08012 (2016)

9. V. Paradiso, K. Amgarou, N. Blanc de Lanaute, F. Bonnet, O. Beltramello, E. Liénard, 3-D localization of radioactive hotspots via portable gamma cameras, Nucl. Instr. Meth. Phys. Res. A 910, 194 (2018)

10. C.M. Whitney, L. Soundara-Pandian, E.B. Johnson, S. Vogel, B. Vinci, M. Squillante, J. Glodo, J.F. Christian, Gamma-neutron imaging system utilizing pulse shape discrimination with CLYC, Nucl. Instrum. Methods Phys. Res. A 784, 346 (2015)

11. C. Lynde, F. Carrel, V. Schoepff, C. Frangville, R. Woo, A. Sardet, J. Venara, M.B. Mosbah, R.A. Khalil, Z. El Bitar, Demonstration of coded-aperture fast-neutron imaging based on Timepix detector, Nucl. Instrum. Methods Phys. Res. A (2018), https://doi.org/10.1016/j.nima.2018.10.051

12. F. Lamadie, F. Delmas, C. Mahé, P. Gironès, C. Le Goaller, J.R. Costes, Remote alpha imaging in nuclear installations: New results and prospects, IEEE Trans. Nucl. Sci. 52, 3035 (2005)

13. NEA, Radiological Characterization for Decommissioning of Nuclear Installations, 2013, available at: https://www.oecdnea.org/rwm/docs/2013/rwm-wpdd2013-2.pdf

Cite this article as: Margarita Herranz, Raquel Idoeta, Khalil Amgarou, Frédéric Aspe, Csilla Csöme, Sven Boden, Marielle Crozet, INSIDER WP5 (in situ measurements): developed activities, main results and conclusions, EPJ Nuclear Sci. Technol. 6, $12(2020)$ 